\title{
Positionspapier der DRG, DGNR, GPR, DeGIR, ÖRG und DGP zur Nutzung klinischer Daten für wissenschaftliche Zwecke
}

Autoren

Christiane Kuhl' ${ }^{1}$ Peter Walter ${ }^{2}$, Claus Zimmer ${ }^{3}$, Hans-Joachim Mentzel ${ }^{4}$, Peter Reimer ${ }^{5}$, Klaus A. Hausegger ${ }^{6}$, Gustavo Baretton ${ }^{7}$, Ralf-Thorsten Hoffmann ${ }^{8}$, Walter Heindel ${ }^{9}$, Christoph Düber ${ }^{10}$, Michael Uder ${ }^{11}$, Konstantin Nikolaou ${ }^{12}$, Gerald Antoch ${ }^{13}$

Vorstand der Deutschen Röntgengesellschaft e. V. (DRG):

Gerald Antoch, Stefan O. Schönberg, Jörg Barkhausen, Frank Anton, Stefan Neumann, Günter Layer, Arnd Dörfler, Friederike Körber, Johannes Weßling, Michael Wucherer

Vorstand der Deutschen Gesellschaft für Neuroradiologie e. V. (DGNR):

Claus Zimmer, Ansgar Berlis, Horst Urbach, Marc A. Brockmann, Mirko Pham, Werner Weber

Vorstand der Gesellschaft für Pädiatrische Radiologie e. V. (GPR):

Hans-Joachim Mentzel, Thekla von Kalle, Dirk Klee, Gabriele Hahn, Janina Patsch

Vorstand der Deutschen Gesellschaft für Interventionelle Radiologie und Minimalinvasive Therapie (DeGIR): Peter Reimer, Marcus Katoh, Ansgar Berlis, Ralf-Thorsten Hoffmann, Peter Landwehr, Andreas Mahnken, Philipp Paprottka

Präsidium der Österreichischen Röntgengesellschaft (ÖRG):

Rosemarie Forstner, Klaus A. Hausegger, Christian Loewe, Helmut Prosch, Gernot Böhm, Elke R. Gizewski, Gerlig Widmann, Gertraud Heinz, Klaus Wicke, Martin Uggowitzer

Vorstand der Deutschen Gesellschaft für Pathologie e. V. (DGP):

Gustavo Baretton, Christoph Röcken, Jörg Maas, Eva Wardelmann, Irene Esposito, Silke Lassmann, Annette Lebeau, Aurel Perren, Andreas Rosenwald, Philipp Ströbel, Wilko Weichert, Sebastian Försch

Konferenz der Lehrstuhlinhaber für Radiologie e. V.:

Gerhard Adam, Gerald Antoch, Ulrike Attenberger, Fabian Bamberg, Jörg Barkhausen, Meinrad Beer, Thorsten Bley, Arno Bücker, Timm Denecke, Christoph Düber, Michael Forsting, Bernd Hamm, Walter Heindel, Ralf-Thorsten Hoffmann, Norbert Hosten, Olav Jansen, Hans-Ulrich Kauczor, Thomas Kröncke, Gabriele A. Krombach, Christiane Kuhl, Joachim Lotz, Andreas Mahnken, David Maintz, Marcus R. Makowski, Konstantin Nikolaou, Maciej Pech, Jens Ricke, Stefan O. Schönberg, Andreas G. Schreyer, Christian Stroszczynski, Ulf Teichgräber, Michael Uder, Thomas J. Vogl, Frank Wacker, Marc-André Weber, Walter A. Wohlgemuth

Institute

1 RWTH Aachen, Medizinische Fakultät, Klinik für Diagnostische und Interventionelle Radiologie, Aachen

2 RWTH Aachen, Medizinische Fakultät, Klinik für Augenheilkunde, Aachen

3 Technische Universität München, Medizinische Fakultät, Abteilung für Diagnostische und Interventionelle Neuroradiologie, München

4 Friedrich-Schiller-Universität Jena, Medinische Fakultät, Institut für Diagnostische und Interventionelle Radiologie, Sektion Pädiatrische Radiologie, Jena

5 Stätisches Klinikum Karlsruhe, Diagnostische und Interventionelle Radiologie, Karlsruhe

6 Klinikum Klagenfurt, Institut für Diagnostische und Interventionelle Radiologie, Klagenfurt

7 Technische Universität Dresden, Medizinische Fakultät Carl Gustav Carus, Institut für Pathologie, Dresden
8 Technische Universität Dresden, Medizinische Fakultät Carl Gustav Carus, Institut und Poliklinik für Diagnostische und Interventionelle Radiologie, Dresden

9 Westfälische Wilhelms-Universität Münster, Medizinische Fakultät, Institut für Klinische Radiologie, Münster

10 Johannes-Gutenberg-Universität Mainz, Medizinische Fakultät, Klinik und Poliklinik für Diagnostische und Interventionelle Radiologie, Mainz

11 Friedrich-Alexander-Universität Erlangen-Nürnberg, Medizinische Fakultät, Radiologisches Institut, Erlangen

12 Eberhard-Karls-Universität Tübingen, Medizinische Fakultät, Diagnostische und Interventionelle Radiologie, Tübingen

13 Heinrich-Heine-Universität Düsseldorf, Medizinische Fakultät, Institut für Diagnostische und Interventionelle Radiologie, Düsseldorf

online publiziert 02.02 .2021 


\author{
Bibliografie \\ Fortschr Röntgenstr 2021; 193: 381-387 \\ DOI 10.1055/a-1352-9747 \\ ISSN 1438-9029 \\ (C) 2021. Thieme. All rights reserved. \\ Georg Thieme Verlag KG, Rüdigerstraße 14, \\ 70469 Stuttgart, Germany
}

Korrespondenzadresse

Prof. Dr. med. Gerald Antoch

Institut für Diagnostische und Interventionelle Radiologie

Universitätsklinikum Düsseldorf, Moorenstr. 5,

40225 Düsseldorf, Deutschland

antoch@med.uni-duesseldorf.de

\section{Hintergrund}

Die zunehmende Verfügbarkeit digitaler Daten, einer leistungsfähigen IT-Infrastruktur sowie die Entwicklung von Methoden, große Datenmengen computergestützt per Algorithmen auszuwerten, werden die medizinische Forschung revolutionieren. In Kombination mit der entsprechenden Hardware ermöglichen Verfahren der sogenannten künstlichen Intelligenz bzw. des maschinellen Lernens die Analyse verschiedenster Datenarten großer Patientenkollektive und bieten so neue Möglichkeiten, ein fach- und datenübergreifendes Verständnis für die Zusammenhänge einzelner Erkrankungen zu generieren. Die Basis für diese zukunftsweisende Forschung ist die Verfügbarkeit eines möglichst vollständigen Datensatzes zum entsprechenden Patientenkollektiv. Daten zur Anamnese, dem klinischen Untersuchungsbefund, Labor, bildgebenden Untersuchungen, feingeweblichen Untersuchungen, dem Krankheitsverlauf usw. müssen fachübergreifend zur Verfügung gestellt werden, um die notwendige Basis für derartige Forschungsansätze zu legen.

Für solche Forschungsfragen muss neben den im Rahmen wissenschaftlicher Projekte, z. B. der „Nationalen Kohorte“, erhobenen Datensätzen in aller Regel auch auf Daten zugegriffen werden, die im Rahmen der regulären Krankenversorgung ambulant oder stationär von verschiedenen Fachabteilungen erhoben werden (im Weiteren als „klinische Daten“ bezeichnet).

Ob die wissenschaftliche Verwendung von Daten, die die Universitätskliniken im Rahmen ihres klinischen Versorgungsauftrages erheben, nur nach jeweils expliziter Einwilligung durch den einzelnen Patienten zu nutzen sind, oder ob sich diese Einwilligung aus dem expliziten Versorgungsauftrag von Universitätskliniken ergibt, ist an anderer Stelle zu diskutieren. Betont sei an dieser Stelle lediglich, dass Universitätskliniken ihrem gesellschaftlich konsentierten Versorgungsauftrag zukünftig nur dann nachkommen können, wenn hier praktikable Lösungen gefunden werden, die das Recht auf informationelle Selbstbestimmung gegenüber dem gesellschaftlichen Nutzen anonymisierter oder pseudonymisierter Datensätze für die klinische Forschung angemessen austarieren.

Der folgende Text befasst sich mit der Frage, nach welchen Regeln klinische Daten für klinische und nichtklinische Wissenschaftler ${ }^{1}$ zur Bearbeitung von Forschungsprojekten verfügbar gemacht werden sollen. Hier besteht erheblicher Regelungsbedarf zunächst im Binnenverhältnis auf Ebene der einzelnen Fakultät,

1 Im Interesse der Lesbarkeit haben wir auf geschlechtsbezogene Formulierungen verzichtet. Selbstverständlich sind immer $w / m$ / d gemeint, auch wenn explizit nur eines der Geschlechter angesprochen wird. nachgeordnet genauso für die Governance von interfakultären Forschungsverbünden sowie von nationalen und schließlich internationalen Vernetzungsinitiativen.

Dieser Regelungsbedarf wird auch im aktuellen Positionspapier des Wissenschaftsrats unterstrichen [1]. Klinische Daten sind ohne jeden Zweifel auch wissenschaftliche Daten im Sinne des Wissenschaftsrats, da sie in einem universitären Umfeld immer auf der wissenschaftlichen Tätigkeit und Expertise des jeweiligen Fachvertreters und/oder der unter seiner Leitung tätigen Wissenschaftler beruhen. In seinem aktuellen Papier geht der Wissenschaftsrat detailliert auf die sogenannte datenintensive Forschung ein und definiert Forderungen für Regelungen des Datenteilens, also eine „Daten-Governance“ - ohne jedoch selbst Regeln zu definieren und damit vorzugeben.

Mit seinem aktuellen Positionspapier versucht der Wissenschaftsrat daher „Aufmerksamkeit auf jene Herausforderungen“ zu lenken, die „über den Auf- und Ausbau neuer Organisationen hinausgehen “, und meint hiermit u. a. die Schaffung von Regeln zum Datenteilen: Er fordert "faires Teilen von Daten in der Wissenschaft" sowie die „Anerkennung der erbrachten Leistungen über disziplinäre, institutionelle und geografische/politische Grenzen hinweg"; dies sei Grundvoraussetzung für die Motivation von Wissenschaftlern, ihre Daten für datenintensive Forschung zur Verfügung zu stellen. Der Wissenschaftsrats empfiehlt, solche Governance-Regeln auf Kooperation und gegenseitige Anerkennung basieren zu lassen.

Zu den vom Wissenschaftsrat in diesem Zusammenhang genannten Organisationen gehört die durch die DFG geförderte Nationale Forschungsdateninfrastruktur (NFDI) [2] des Bundes und der Länder, die das Ziel verfolgt, wissenschaftliche Daten national und international zugänglich zu machen. Hierfür werden verschiedene Konsortien gefördert, die aus „Zusammenschlüssen von Nutzenden und Anbietenden von Forschungsdaten” bestehen, die „auf langfristige Zusammenarbeit” angelegt sind, und „Hochschulen, auBeruniversitäre Forschungseinrichtungen, Ressortforschungseinrichtungen, Akademien und andere öffentlich geförderte Informationsinfrastruktureinrichtungen" umfassen. Die NFDI sieht die Anbietenden von Forschungsdaten in einer zentralen Rolle innerhalb der geförderten Konsortien. Wie der Wissenschaftsrat jedoch feststellt, steht bei der NFDI die Schaffung von Infrastruktur für die Vernetzung im Vordergrund. Die Aufstellung von Regeln, wie denn konkret innerhalb der Konsortien die Rechte des Datenzugriffs verteilt werden sollen, wird dagegen den einzelnen Konsortien selbst überlassen. Auch hier bedarf es daher einer klaren Regelung des Datenzugriffs auf lokaler Ebene, die die Vorgaben der guten wissenschaftlichen Praxis und alle rechtlichen Vorgaben berücksichtigt. 
Ein weiteres großes Verbundprojekt ist die vom Bundesministerium für Bildung und Forschung geförderte MedizininformatikInitiative [3]. Diese hat zwar Vorschläge für Regelungen der Daten-Governance im Rahmen großer Konsortialprojekte entwickelt; so wurden ein Eckpunktepapier für eine einheitliche Nutzungsordnung [4] und eine Muster-Nutzerordnung [5] entwickelt, die den einzelnen Konsortien als Vorlage dienen können. Regelungen der Daten-Governance auf Ebene des einzelnen Konsortialpartners fehlen jedoch bislang. Solche Regeln sind jedoch wesentlich, um die Interessen der lokalen Datenerzeuger zu berücksichtigen.

Regelungen für das Teilen von Daten bzw. einer Daten-Governance fehlen bislang also auf lokaler Ebene, d. h. innerhalb einer Fakultät, und sind auch für die großen nationalen Vernetzungsinitiativen noch nicht vervollständigt.

Eine juristische Regelung des Zugriffs auf klinische Daten ist überaus komplex, da sie Vorgaben aus den Bereichen des Urheber-, des Eigentums-, des Verwertungs- und des Datenschutzrechts unterliegen würde. Diese juristischen Vorgaben bilden bei der Diskussion um Zugriffs- und Nutzungsrechte zweifelsohne die rechtliche Basis, die uneingeschränkt berücksichtigt werden muss. Allerdings ist das bestehende juristische Regelwerk wenig geeignet, die Zugriffsrechte der verschiedenen Zugriffsinteressenten innerhalb einer Fakultät, zwischen Medizinischer Fakultät und Klinikum bzw. den verschiedenen Fakultäten einer Universität oder Hochschule, oder zwischen verschiedenen Hochschulstandorten zu regeln. Dies birgt erhebliches Konfliktpotenzial. Mit dem deutlich steigenden Interesse an datengetriebener Wissenschaft und den Methoden des maschinellen Lernens sowie in Anbetracht des stetig steigenden Publikationsdrucks, der die Entscheidungswege innerhalb der Fakultäten zunehmend prägt, steigt die Wahrscheinlichkeit von innerfakultären Auseinandersetzungen über Datenzugriffsmöglichkeiten. Diese Konflikte können den Fakultätsfrieden und das produktive Miteinander einer Fakultät empfindlich stören.

Um bei der Frage einer Nutzung klinischer Daten durch andere Wissenschaftler als dem Datenerzeuger zu einer Lösung zu kommen, legen die Verfasser dieses Artikels eine auf den Grundlagen der guten wissenschaftlichen Praxis sowie den Empfehlungen des Wissenschaftsrats basierende Regelung vor, die die Nutzung klinischer Daten interdisziplinär, intra- und interfakultär regelt. Basis einer solchen Regelung sollte immer eine wertschätzende, auf Kooperation der verschiedenen wissenschaftlichen Partner ausgelegte Vereinbarung sein, wie sie beispielsweise an der Medizinischen Fakultät der Rheinisch-Westfälischen Technischen Hochschule Aachen nach fakultätsinterner Diskussion erarbeitet und implementiert wurde [6].

\section{Gegenstand}

Für alle in der klinischen Patientenversorgung involvierten Personen gilt gleichermaßen, dass die von ihnen erzeugten klinischen Daten im Rahmen ihres vertraglich festgelegten Dienst- und Versorgungsauftrags erhoben werden, wofür sie durch Fakultät und Klinikum vergütet werden. Uneingeschränktes Zugriffsrecht auf die erzeugten klinischen Daten besteht nur zum Zwecke der indi- viduellen Krankenversorgung eines einzelnen Patienten. Das Recht zur weiterführenden Nutzung klinischer Daten für Forschungszwecke und damit auch einhergehend die gleichzeitige Verpflichtung zu einem verantwortungsvollen Umgang mit diesen Daten liegt bislang ausschließlich bei dem Lehrstuhlinhaber, in dessen Verantwortungsbereich diese Daten erzeugt wurden und der das explizite Mandat seiner Fakultät hält, sein Fach und damit die in seinem Fach erzeugten Daten wissenschaftlich zu vertreten. Diese unterschiedlichen Zugriffsrechte bezogen auf die Nutzung klinischer Daten zeigt die juristische Komplexität des Sachverhalts.

Entsprechend ist im Folgenden keine juristische Erörterung oder Bewertung der Frage der Datennutzung vorgesehen. Vielmehr geht es darum, gemeinsame Regeln für den einvernehmlichen, fairen Umgang mit klinischen Daten zu finden.

\section{Zugrunde gelegtes Begriffsverständnis}

Ziel einer Regelung der Datennutzung muss die Förderung einer kooperativen Forschung sein. „Kooperativ“ ist die wissenschaftliche Tätigkeit dann, wenn Vertreter verschiedener Einrichtungen gemeinsam eine wissenschaftliche Fragestellung bearbeiten und dabei alle zum Endergebnis beitragen. Die Beantragung einer Forschungsförderung, die Auswertung und die Publikation der Ergebnisse erfolgen gemeinsam, alle Beteiligten profitieren von dieser kooperativen wissenschaftlichen Tätigkeit. Ebenso profitieren auch die Forschungsprojekte inhaltlich durch kooperative Interdisziplinarität. Die Notwendigkeit einer kooperativen Bearbeitung wissenschaftlicher Projekte wird vom Wissenschaftsrat explizit hervorgehoben [1].

Im Gegensatz dazu ist Forschung „nichtkooperativ“, wenn Daten ohne Berücksichtigung ihrer Urheberschaft Dritten für wissenschaftliche Analysen zur Verfügung gestellt werden, ohne dass ein direkter, für alle Beteiligten sicht- oder messbarer Mehrwert entsteht.

Eine Datennutzung durch Dritte für Forschung zum Schaden des Datenerzeugers ist schließlich „parasitär“ - ein Beispiel hierfür ist die Publikation von Daten des Datenerzeugers durch Dritte, die dem Datenerzeuger selbst die Möglichkeit nimmt, seine selbst erzeugten Daten selbst zu publizieren.

Bei der Regelung des Umgangs mit klinischen Daten muss zwischen der „Datenerzeugung“ und der „Datenaufbereitung“ oder „Datenkuratierung“ differenziert werden:

Derjenige, der die Daten erhebt, ist der „Erzeuger“ im Sinne des u. g. „Erzeugerprinzips“ und Inhaber des Erstverwendungsrechts. Alle anderen Tätigkeiten zählen zur „Aufbereitung“. Gemäß dieser Definition entspricht beispielsweise die Digitalisierung vorhandener Arztbriefe oder die Bearbeitung solch digitalisierter Arztbriefe mit den Methoden des natural language processings zum Zwecke der automatisierten Textanalyse einer „Aufbereitung“ vorhandener Daten. Die Erzeugerschaft dieser Daten liegt aber immer noch bei dem den Arztbrief abfassenden Arzt. Zur „Aufbereitung“ von Daten zählt auch die Kumulation von Daten verschiedener Herkunft in einer multidisziplinären Datenbank.

„Erzeuger“ der Daten ist somit der diese Daten im Rahmen seiner wissenschaftlichen oder klinischen Tätigkeit generierende 
Lehrstuhlinhaber, der das jeweilige Fach in Forschung und Lehre vertritt und der seine nach- oder beigeordneten Mitarbeiter im Außenverhältnis vertritt.

An dieser Tatsache ändert auch eine mögliche Vergütung im Innenverhältnis der Krankenversorgung nichts. Das im Rahmen der Krankenversorgung durchgeführte Thorax-CT, das im Rahmen einer internen Leistungsverrechnung dem Zuweiser in Rechnung gestellt wird, wird vom Erzeuger nicht „verkauft“ und vom Zuweiser nicht „erworben“. „Erzeuger“ des Thorax-CT bleibt daher der Radiologe. Kurz: Es erfolgt kein „Abtreten“ des Erzeugerstatus, wenn die Datenerzeugung z. B. per interner Leistungsverrechnung oder durch Dritte vergütet worden ist.

In Datenbanken für datenintensive Forschung ist eine „Kuratierung“ von Daten erforderlich, um sie sinnstiftend bzw. optimal wissenschaftlich nutzen zu können. Diese Kuratierung erfordert in aller Regel noch einmal einen zusätzlichen Beitrag durch den Datenerzeuger - denn nur dieser ist in der Lage, die erforderliche Charakterisierung und Aktualisierung der eingespeisten Daten sachgerecht durchzuführen. Dies ist als besonderer zusätzlicher Beitrag des Datenerzeugers, also Fachvertreters, zu würdigen. So stellt der Wissenschaftsrat fest: Für die Kuratierung von Daten ist „...häufig noch eine aufwendige Mitwirkung“ des Datenerzeugers erforderlich [1]. In Leitlinie 4 weist der Wissenschaftsrat explizit auf die anzuerkennende Leistung der Wissenschaftler hin: „Hochwertige Beiträge zu kuratierten Datensammlungen und Datenpublikationen, zum Forschungsdatenmanagement wie auch zur Methoden- und Softwareentwicklung müssen als genuine Leistungen von Wissenschaftlerinnen und Wissenschaftlern anerkannt werden und Unterstützung erfahren.“ [1]

\section{Optionen zum Umgang mit klinischen Daten für wissenschaftliche Zwecke}

Als Regel für die Zuordnung von Verwendungsrechten zur Nutzung klinischer Daten kommen 2 grundsätzliche Vorgehensweisen in Betracht: Das Zugriffsprinzip und das Erzeugerprinzip.

\section{(A) Zugriffsprinzip}

Grundannahme des Zugriffsprinzips ist, dass klinische Daten niemandem „gehören“ und damit für die wissenschaftliche Verwendung grundsätzlich für alle Interessierten verfügbar sind bzw. gemacht werden müssen. Jeder Interessierte kann auf Daten zugreifen; der Erzeuger ist (allenfalls) zu informieren. Der Erzeuger kann ein konstruktives Veto einlegen, d. h. kann ein anderes Vorgehen vorschlagen - muss aber grundsätzlich kooperieren, d. h. seine Daten jedem interessierten Dritten, auch außerhalb der von ihm verantworteten wissenschaftlichen Arbeitsgruppen, zur Verfügung stellen. Andernfalls muss er ein nachvollziehbares eigenes Interesse an der Verwertung belegen. Im Falle einer Auseinandersetzung entscheidet ein zu wählendes, paritätisch besetztes Gremium oder der Vorstand der jeweiligen Einrichtung über den „Zuschlag“, wobei Kriterien für die Entscheidungen dieses Gremiums zu entwickeln wären.

\section{(B) Das Erzeugerprinzip}

Grundannahme des Erzeugerprinzips ist, dass der Erzeuger von klinischen Daten ein Erstverwendungsrecht auf diese Daten hält und über deren wissenschaftliche Verwendung primär bestimmt. Vorbild des Erzeugerprinzips sind die Leitlinien zur Sicherung guter wissenschaftlicher Praxis der Deutschen Forschungsgemeinschaft: „Die Nutzung steht insbesondere der Wissenschaftlerin und dem Wissenschaftler zu, die/der sie erhebt. Im Rahmen eines laufenden Forschungsprojekts entscheiden auch die Nutzungsberechtigten (insbesondere nach Maßgabe datenschutzrechtlicher Bestimmungen), ob Dritte Zugang zu den Daten erhalten sollen” [7]. Beispielhaft können aber auch aktuell gültige Regeln, wie sie für den Betrieb vieler Biobanken gelten, angeführt werden: Der Erzeuger von klinischen Daten wie der Einsender von organischen Gewebeproben, die im Rahmen der „Routineversorgung“ erhoben und ggf. in Datenbanken eingegliedert werden, behält das natürliche Erstverwendungsrecht für diese Daten (Proben). Damit ist auch die Verpflichtung verbunden, die Daten vor Missbrauch oder nicht adäquater Verwendung zu schützen und andererseits aber auch für kooperative Forschung zur Verfügung zu stellen.

\section{Vorschlag der beteiligten Fachgesellschaften zur Regelung}

Die Verfasser sprechen sich klar für Vorgehen (B), das Erzeugerprinzip, aus: Der Erzeuger von klinischen Daten regelt den Zugriff Dritter auf die von ihm erzeugten klinischen Daten. Dritte, die Zugriff auf Daten des Erzeugers erhalten wollen, setzen sich mit dem Erzeuger direkt ins Benehmen. Im Rahmen kooperativer Forschungsprojekte wird der Erzeuger von Daten in der Regel einen Mehrwert in der gemeinsamen Bearbeitung von Fragestellungen sehen und entsprechend seine Daten bereitstellen.

Der Erzeuger von Daten hält also das natürliche (primäre) Nutzungsrecht oder „Erstverwendungsrecht“ an seinen Daten. Das Erstverwendungsrecht bezieht sich dabei auf die Verwendung klinischer Daten für sämtliche wissenschaftliche Fragestellungen. Hat beispielsweise ein Erzeuger seine Daten im Hinblick auf eine Fragestellung ausgewertet, so behält er das Erstverwendungsrecht auch zur Auswertung derselben Daten für weitere wissenschaftliche Fragestellungen. Das Erstverwendungsrecht setzt selbstverständlich voraus, dass der Erzeuger die Daten auch wissenschaftlich nutzen will und wird. Ist dies nicht der Fall, besteht die Verpflichtung, die Daten interessierten Nutzern für die wissenschaftliche Auswertung zur Verfügung zu stellen. Die adäquate Nennung der Datenquelle ist in diesem Fall jedoch zwingend erforderlich.

\section{Begründung}

Dass kooperative klinische Forschung seit Jahrzehnten in der deutschen Universitätsmedizin mehr oder weniger geräuschlos gelingt und in den vergangenen Jahren erheblich intensiviert worden ist, liegt u. a. daran, dass die Regeln des Miteinanders, wenn auch bis- 
lang nicht verschriftlicht, doch einfach und klar gelebt werden: Der Erzeuger von Daten hält das natürliche (primäre) Nutzungsrecht; auch wenn er Daten oder Gewebeproben in eine (Bio-) Datenbank einsendet, behält er die Urheberschaft und damit das primäre Nutzungsrecht. Die bisher gelebte Regel entspricht damit im Kern den für die meisten Biobanken verschriftlichten Regeln. Sie entspricht zudem der Regel über „data ownership“ des von Europäischen und US-Amerikanischen Fachgesellschaften herausgegebenen White Papers zu „Ethics in Al“ [8].

Auch der Wissenschaftsrat fordert in Leitlinie 1 des Positionspapiers, dass Regelungen derart gestaltet sein müssen, „dass sie das Teilen von Daten und Software in der Wissenschaft und ihre kooperative Bearbeitung fördern“, und präzisiert dies: „Zugangsbeschränkungen zu in der Wissenschaft erzeugten oder bearbeiteten Daten ..." können gemäß Feststellung des Wissenschaftsrats „... nicht nur aus rechtlichen Gründen notwendig sein“ sondern gerade auch, um kooperative Forschung sicherzustellen [1].

Das Zugriffs- und Verwendungsrecht von klinischen Daten für Wissenschaft ist also zu regeln. Bestehende rechtlich-juristische Regelwerke sind wenig geeignet, dieses Zugriffs- und Verwendungsrecht zu klären, weil Begrifflichkeiten des Urheber- oder Eigentumsrechts kaum sinnstiftend anzuwenden sind oder ihre Anwendung mit großen rechtlichen Risiken für die beteiligten Wissenschaftler, Fakultäten und Forschungsinitiativen verbunden ist - was auch erklärt, warum solche Regeln bislang weitestgehend fehlen.

Wir schlagen daher eine Regel vor, die sich bewusst nicht aus juristischen Ansprüchen ableiten lässt, sondern einer freiwilligen Festlegung entspricht; einem „Comment“, der für den Erhalt des Fakultätsfriedens und des gemeinsamen, also kooperativen wissenschaftlichen Arbeitens erforderlich ist und daher für alle Beteiligten bindend sein sollte. Unser Vorschlag ist, das primäre Nutzungs- und Verwendungsrecht beim Datenerzeuger ${ }^{2}$ zu verorten. Von allen möglichen Festlegungen ist dies die plausibelste und pragmatischste, die auch der bisher gelebten, wenn auch nicht verschriftlichten Praxis entspricht.

Die Festlegung, das Verwendungsrecht klinischer Daten dem Erzeuger zuzuordnen und das Prinzip des „freien Zugriffs“ nicht zu verfolgen, lässt sich inhaltlich - nicht juristisch - wie folgt begründen:

a) Dass das Nutzungsrecht primär beim Erzeuger von Daten liegt, ist zur Qualitätssicherung der Forschung essenziell, da der Erzeuger der Daten die dafür notwendige Expertise bezüglich Datenerhebung, Datenaufbereitung und Datenauswertung bietet.

b) Bedenken gegen den freien Zugriff auf klinische Daten bestehen insofern, als die Erzeuger klinischer Daten kraft ihrer individuellen fachlichen Qualifikation einen qualitätssichernden Input nicht nur bei der Interpretation klinischer Daten leisten, sondern auch bei deren Zustandekommen. Der persönliche Beitrag des Fachvertreters oder von Mitarbeitern seiner Abteilung ist maßgeblich für die Qualität der erzeugten Daten. Dieser Beitrag besteht z. B. in der Festlegung des je nach Patienten

2 Der Datenerzeuger ist der Fachvertreter, stellvertretend für die Mitarbeiter im von ihm vertretenen Verantwortungsbereich und Fragestellung geeigneten Untersuchungsgangs oder -protokolls oder in der Festlegung einer bestimmten Interventions-, Endoskopie- oder Operationstechnik. Bereits die Erzeugung klinischer Daten wird also durch den persönlichen Beitrag des jeweiligen Fachvertreters entscheidend geprägt. So ist auch erklärt, dass nicht nur qualitätsgesicherte Primärdaten, sondern bereits die Rohdaten dem hier diskutierten Erstverwendungsrecht des Erzeugers unterliegen.

c) Der Fachvertreter erhält mit seiner Berufung explizit und implizit das Mandat der Fakultät, die von ihm erhobenen klinischen Daten - also Daten seines Fachgebiets - wissenschaftlich zu verwerten. Bei Umsetzen des Zugriffsprinzips wird dem Erzeuger von Daten auferlegt, sein „eigenes Interesse“ an den Daten zu belegen. Dieses Vorgehen ist mit der Freiheit in Forschung und Lehre nicht vereinbar. Für den Fachvertreter wird es schwierig sein, ein entscheidungsbefugtes Ombudsgremium davon zu überzeugen, dass er mit den Daten selbst hätte wissenschaftlich arbeiten wollen. Seine Position als Fachvertreter und als Erzeuger der Daten wird im Kontext des Zugriffsprinzips demnach nicht mehr als ausreichend angesehen, ein eigenes Verwertungsinteresse zu belegen. Dies entspricht de facto einer Beweislastumkehr im Vergleich zum Erzeugerprinzip. Es entspricht zudem einem Entzug des Mandats, das eigene Fach durch wissenschaftliche Nutzung selbst erzeugter Daten wissenschaftlich zu vertreten. Langwierige rechtliche Auseinandersetzungen zwischen Fakultät und Fachvertretern sind hier zu befürchten.

d) Für die Umsetzung des Zugriffsprinzips müsste anstelle der guten wissenschaftlichen Praxis ein komplexes Regelwerk neu entwickelt werden, das definieren müsste, zu welchen Bedingungen, unter welchen Prämissen und zu welchen Konditionen Daten an Dritte für Forschungsprojekte zur Verfügung gestellt werden müssen. Die Definition eines solchen komplexen Regelwerks ist unter Berücksichtigung der verschiedenen rechtlichen Aspekte mit großen juristischen Risiken verbunden. Ein Regelwerk basierend auf dem Zugriffsprinzip fördert nicht, sondern verhindert die flexible Kooperation mehrerer Partner miteinander und ist mit den Leitlinien der guten wissenschaftlichen Praxis der DFG nicht vereinbar.

e) Auch die Entscheidung von Konfliktfällen durch ein Ombudsgremium ist problematisch, da es die Regelung von Streitfällen auf der Basis eines Mehrheitsbeschlusses durchsetzen würde. Jedoch ist der Erzeuger von Daten per se stets in einer Minderheitsposition. Alle anderen Fakultätsmitglieder außer dem Datenerzeuger selbst werden im Allgemeinen immer einen Vorteil darin sehen, besagte Daten für andere verfügbar zu machen; aus ebendiesem Grund wird in der deutschen Gesetzgebung „Urheberschutz“ - als Schutz der Minderheit als wichtiges Rechtsgut angesehen. Es wird darüber hinaus schwierig zu definieren, aufgrund welcher Kriterien das Ombudsgremium entscheiden soll (siehe Punkt g). Langwierige rechtliche Auseinandersetzungen sind auch hier vorprogrammiert und nicht im Interesse der Wissenschaft und der Hochschulen.

f) Die Zugriffsregelung entspräche einer Aufkündigung der bisher gelebten Praxis. Fachvertreter wären zukünftig gezwungen, ihre Daten Dritten zur Verfügung zu stellen, die ihrerseits 
möglicherweise keine klinischen Daten erzeugen und keinen für den Erzeuger der Daten erkennbaren nutzbringenden Beitrag leisten. Da beim Zugriffsprinzip interessierte Dritte, die Daten nutzen wollen, nicht mehr auf die Zustimmung des Datenerzeugers angewiesen sind bzw. seinen Widerspruch durch Anrufung eines Ombudsgremiums umgehen können, hätten Datennutzungsinteressenten zukünftig auch keine Veranlassung mehr, für den Datenerzeuger einen Mehrwert zu erzeugen oder diesen überhaupt zu involvieren. Dies fördert kooperative Datennutzung nicht nur nicht - sondern untergräbt das ihr zugrunde liegendes Wirkprinzip, nämlich die Kooperation.

g) Wenn Daten aus der Patientenversorgung frei zugänglich gemacht werden sollen, könnte sich schließlich ein Wettbewerbsnachteil ergeben für wissenschaftlich tätige Mediziner, deren Aufgabe es ist, ebendiese Daten im Rahmen ihrer klinischen Verpflichtungen zu erzeugen, gegenüber Wissenschaftlern aus Instituten ohne Patientenversorgung. Wissenschaftler aus Instituten ohne Patientenversorgung könnten durch Definition einer Vielzahl möglicher Fragestellungen und Projekte vielfältige thematische Pflöcke einschlagen, sodass die Datenerzeuger, die mit der Erzeugung ebendieser Daten u. U. tagesfüllend beschäftigt sind, im Nachteil sind, da sie kaum werden belegen können, dass sie - hätten sie die Zeit dazu gehabt dieselben Projekte auf den von ihnen erzeugten Daten hätten durchführen wollen.

h) Der aktuelle Zeitgeist bewertet das Recht auf freien Zugang zu Daten teils höher als das Recht des Urhebers an seinen selbst erzeugten Daten. Dies zeigt sich u. a. an der Diskussion rund um das Urheberrecht zu im Internet veröffentlichten Texten, Fotos und Filmen („Netzfreiheit“). Wenn sich die Rechtsauffassung - dem Zeitgeist folgend - zukünftig auf die Seite derjenigen stellen sollte, die freien Zugriff auf solche Daten fordern, so ist diesbezüglich klar zu konstatieren, dass die im Netz sichtbaren Inhalte in aller Regel vom Erzeuger selbst und freiwillig eingestellt worden sind. Die aktuelle Entscheidung des EU-Parlaments (EU-Urheberrechtreform) hat nun erneut und eindeutig die Rechte der Erzeuger von Inhalten gestärkt.

\section{Eckpunkte des Erzeugerprinzips}

Kooperative Forschung unter Anwendung des Erzeugerprinzips kann durch folgende Eckpunkte sichergestellt werden [6]:

1. Die wissenschaftliche Nutzung der eigenen Daten durch den Erzeuger ist mit einem Veto-Recht des Erzeugers gegen die Nutzung der Daten durch Dritte verbunden. Ein Veto bedarf der Begründung entsprechend der Empfehlungen des Wissenschaftsrats [1] und ist nicht überstimmbar.

2. Beansprucht oder nutzt der Erzeuger die selbst erzeugten Daten nicht für eine eigene wissenschaftliche Auswertung, stellt er die Daten interessierten Dritten zur Verfügung. Die Auswertung erfolgt dann kooperativ, d. h. unter Einbindung des Erzeugers. Bringt sich der Erzeuger nicht weiter in die Auswertung der durch ihn bereitgestellten Daten ein, so ist entsprechend der Regeln guter wissenschaftlicher Praxis zumindest die Nennung des Datenerzeugers notwendig.
3. Für den Konfliktfall kann ein Ombudsgremium einberufen werden, das beratenden Charakter für beide Seiten hat, um eine Einigung zwischen den Parteien zu finden. Dem Gremium sollten Vertreter der klinischen Fächer und des Dekanats angehören.

4. Die datenbereitstellenden Lehrstuhlinhaber sind bei interfakultären Kooperations- und Konsortialverträgen in die Ausgestaltung dieser Verträge einzubeziehen. Der Charakter einer kooperativen Forschung nach dem Erzeugerprinzip muss auch hier gewahrt werden.

5. Unter Berücksichtigung o.g. Rechte des primären Erzeugers von Daten ist anzustreben, dass intra- und interfakultäre Regelungen zur Schaffung von gemeinsamen Datenbanken verschiedener Kliniken und Institute geschaffen werden.

\section{Zusammenfassung}

Kooperative Forschung gelingt, wenn die verschiedenen Fachrichtungen für die gemeinsame Datennutzung aufeinander angewiesen sind, also weiterhin Anlass besteht, eine „win-win“-Situation herzustellen. Dies führt dazu, dass sich die verschiedenen Fachrichtungen zu beiderseitigem Nutzen zu einer Arbeitsgruppe zusammenschließen und eine gemeinsame Verwertungsstrategie erarbeiten. Das „Erzeugerprinzip“ bietet hierfür eine optimale Voraussetzung, fördert kooperative Forschung und vermeidet langwierige rechtliche Auseinandersetzungen. Eine komplett freie Zugriffsregelung hingegen fördert nichtkooperative, ggf. sogar parasitäre Forschung, kann klinisch tätige Fächer benachteiligen und mit großen rechtlichen Risiken verbunden sein. Zudem birgt ein komplett freier Datenzugang die Gefahr des Datenmissbrauchs und des nichtadäquaten Umgangs mit diesen Daten.

Es steht außer Frage, dass fehlende Bereitschaft zur Kooperation im Rahmen wissenschaftlicher Projekte nicht zu akzeptieren ist. Jeder Erzeuger von Daten hat die Verpflichtung, kooperative Forschung zu ermöglichen. Allerdings ist es - nicht nur aus rechtlicher Sicht - kontraproduktiv zu versuchen, den Erzeugern klinischer Daten das Recht zu entziehen, letztlich entscheiden zu können, was mit ihren Daten geschieht. Dieses Recht ist natürliche Voraussetzung für kooperative interdisziplinäre Forschung, die einen Mehrwert für die beteiligten Wissenschaftler generiert. Damit ist dieses Recht letztlich auch die Grundlage für die Integrität der Fächer in Forschung, Lehre und Krankenversorgung.

\section{Interessenkonflikt}

Die Autoren geben folgende Interessenkonflikte an:

Kuhl: Keine Interessenkonflikte

Walter: Keine Interessenkonflikte

Zimmer: Zimmer gab keine relevanten Konflikte in Bezug auf die Aktivi-

täten im Zusammenhang mit diesem Artikel an. Er war in wissenschaftlichen Beiräten für Philips und Bayer Schering tätig. fungiert als Mitherausgeber im Beirat für klinische Neuroradiologie; hat Sprecherhonorare von Bayer-Schering und Philips erhalten; Das Institut erhielt Forschungsunterstützung und Prüfgebühren für klinische Studien von Biogen Idec, Quintiles, MSD Sharp \& Dome, Boehringer Ingelheim, Advance Cor, Brainsgate, Pfizer, Bayer-Schering, Novartis, Roche, Servier. Penumbra, WCT GmbH, Syngis, SSS International Clinical Research, PPD Deutschland GmbH, Worldwide Clinical Trials Ltd., Phenox, Covidien, Actelion, Medivation, 
Medtronic, Harrison Clinical Research, Konzentrisch, Pharmtrace, Reverse Medical Corp., Premier Research Germany Ltd. , Surpass Medical Ltd., GlaxoSmithKline, AXON Neuroscience, Bristol-Myers Squibb, Genentech, Acandis, EISAI, NeuroRx, Italfarmaco, Bioclinica, MIAC und IXICO. Keine Patente erteilt und angemeldet. Derzeit ist er Präsident der Deutschen Gesellschaft für Neuroradiolohy (DGNR).

Mentzel: Mitglied Deutsche Röntgengesellschaft, Mitglied und Präsident Gesellschaft für Pädiatrische Radiologie, Mitglied Deutsche Gesellschaft für Ultraschall in der Medizin

Reimer: Keine Interessenkonflikte

Hausegger: Keine Interessenkonflikte

Baretton: Keine Interessenkonflikte

Hoffmann: Keine Interessenkonflikte

Heindel: Keine Interessenkonflikte

Düber: Keine Interessenkonflikte

Uder: Leiter der Fortbildungsakademie der Deutschen Röntgengesell-

schaft

Nikolaou: Keine Interessenkonflikte

Antoch: Präsident der Deutschen Röntgengesellschaft

\section{Literatur}

[1] Zum Wandel in den Wissenschaften durch datenintensive Forschung; Positionspapier des Wissenschaftsrats. 2020. abrufbar unter https://www.wissenschaftsrat.de/download/2020/8667-20.pdf?__blob= publicationFile \& $=5$

[2] https://www.dfg.de/foerderung/info_wissenschaft/2020/info_ wissenschaft_20_29/index.html

[3] https://www.medizininformatik-initiative.de/de/start

[4] Eckpunktepapier einer einheitlichen Nutzungsordnung der AG DataSharing der Medizininformatik-Initiative. abrufbar unter https://www. medizininformatik-initiative.de/sites/default/files/inline-files/MII_03_ Eckpunktepapier_Nutzungsordnung_1-0.pdf

[5] Übergreifende Muster-Nutzerordnung zum Austausch von Patientendaten, Biomaterialien, Analysemethoden und -routinen im Rahmen der Medizininformatik-Initiative. abrufbar unter https://www.medizininfor matik-initiative.de/sites/default/files/2019-07/MII_Nutzungsordnung_ v1.02_MII_WebSite.pdf

[6] Regelung der Datenverwendung und des Datenzugriffs für Forschungsprojekte am UKA. abrufbar unter https://www.medizin.rwth-aachen.de/ global/show_document.asp?id=aaaaaaaaavwcwhw

[7] Leitlinien zur Sicherung guter wissenschaftlicher Praxis der Deutschen Forschungsgemeinschaft 2019. Kodex. Erläuterungen zu Leitlinie 10, S. 16-17. abrufbar unter https://www.dfg.de/download/pdf/foerderung/ rechtliche_rahmenbedingungen/gute_wissenschaftliche_praxis/ kodex_gwp.pdf

[8] Geis JR et al. Ethics of Artificial Intelligence in Radiology: Summary of the Joint European and North American Multisociety Statement. Radiology 2019; 293 (2): 436-440 